\title{
Association of COPD candidate genes with computed tomography emphysema and airway phenotypes in severe COPD
}

\author{
W.J. Kim**\#, E. Hoffman ${ }^{\star}$, J. Reilly ${ }^{+}$, C. Hersh*^, D. DeMeo*, , \\ G. Washko ${ }^{\S}$ and E.K. Silverman*, ${ }^{*}$
}

ABSTRACT: The principal determining factors influencing the development of the airway disease and emphysema components of chronic obstructive pulmonary disease (COPD) have not been clearly defined. Genetic variability in COPD patients might influence the varying degrees of involvement of airway disease and emphysema. Therefore, we investigated the genetic association of single nucleotide polymorphisms (SNPs) in COPD candidate genes for association with emphysema severity and airway wall thickness phenotypes.

Polymorphisms in six candidate genes were analysed in 379 subjects of the National Emphysema Treatment Trial (NETT) Genetics Ancillary Study with quantitative chest computed tomography (CT) data. Genetic association with per cent of lung area below -950 HU (LAA950), airway wall thickness, and derived square root wall area (SRWA) of 10-mm internal perimeter airways were investigated.

Three SNPS in EPHX1, five SNPs in SERPINE2 and one SNP in GSTP1 were significantly associated with LAA950. Five SNPs in TGFB1, two SNPs in EPHX1, one SNP in SERPINE2 and two SNPs in ADRB2 were associated with airway wall phenotypes in NETT.

In conclusion, several COPD candidate genes showed evidence for association with airway wall thickness and emphysema severity using $\mathrm{CT}$ in a severe COPD population. Further investigation will be required to replicate these genetic associations for emphysema and airway wall phenotypes.

KEYWORDS: Airway, chronic obstructive pulmonary disease, computed tomography, emphysema, genetic association

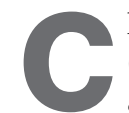

hronic obstructive pulmonary disease (COPD) is characterised by persistent airflow obstruction [1]. Cigarette smoking is a well-known risk factor, but other environmental and genetic factors are likely to be involved. The relative amount of airway and airspace involvement is heterogeneous in COPD subjects, but the key determinants of these different phenotypes, which largely develop from exposure to cigarette smoke, are not known.

Computed tomography (CT) is a useful way of characterising structural changes, including quantitative measurement of emphysema and airway wall phenotypes [2]. A recent paper suggested that genetic components influencing airway wall thickness and emphysema phenotypes were independent [3]. There are several reports of genetic associations with emphysema severity or emphysema distribution [4-6]. However, combined genetic investigation of both emphysema and airway wall phenotypes in the same population has not been widely reported.

We hypothesised that different genetic variants would influence airway disease and emphysema. We investigated whether single nucleotide polymorphisms (SNPs) in previously reported COPD susceptibility genes were associated with emphysema severity and airway wall phenotypes in a COPD study population. We selected six candidate genes that had been associated with COPD susceptibility in at least two populations.

The National Emphysema Treatment Trial (NETT) is a cohort of emphysema subjects with available chest CT data. We measured airway

This article has supplementary material available from www.erj.ersjournals.com

AFFILIATIONS

${ }^{*}$ Channing Laboratory,

${ }^{\mathrm{s}}$ Division of Pulmonary and Critical Care Medicine, Brigham and Women's Hospital, Boston, MA, "Dept of Radiology, University of lowa, lowa City, IA, and

'Division of Pulmonary and Critical Care Medicine, University of Pittsburgh, Pittsburgh, PA, USA. \#Dept of Internal Medicine, Kangwon National University, Chuncheon Korea.

CORRESPONDENCE

E.K. Silverman

Channing Laboratory Brigham and Women's Hospital 181 Longwood Avenue Boston

MA

02115 USA

E-mail: ed.silverman@ channing.harvard.edu

Received

Nov 012009

Accepted after revision:

April 282010

First published online:

June 042010 
wall phenotypes using CT scans in a subset of subjects and investigated associations of variants in COPD susceptibility genes with both emphysema severity and airway wall phenotypes in emphysema subjects from NETT.

\section{METHODS}

\section{Subjects}

The current analysis included 379 non-Hispanic white subjects in the NETT Genetics Ancillary Study with quantitative chest CT data. Subjects enrolled in NETT had severe airflow obstruction (forced expiratory volume in $1 \mathrm{~s}$ (FEV1) $\leqslant 45 \%$ predicted), hyperinflation on pulmonary function testing and bilateral emphysema on high-resolution chest CT [7]. The study was approved by institutional review boards at participating centres. All subjects provided written informed consent.

\section{Chest CT analysis}

Emphysema measurements on NETT subjects have been described previously [6]. CT images of the chest were acquired at full inspiration with a minimum of $200 \mathrm{mAs}$ and reconstructed using a high spatial frequency algorithm with a 1-2$\mathrm{mm}$ collimation at $20-\mathrm{mm}$ intervals. Densitometric measures of emphysema were performed using the Pulmonary Analysis Software Suite (PASS, University of Iowa, Iowa City, IA, USA) at a threshold of $-950 \mathrm{HU}$. Airway wall thickness and the square root of wall area (SRWA) were assessed using 3D Slicer (www.slicer.org) and Airway Inspector (www.airwayinspector.org) at Brigham and Women's Hospital (Boston, MA, USA). The full width at half-maximum method was used to measure the wall thickness and wall area of each airway. From these measures, the airway wall thickness and the SRWA of a 10-mm luminal perimeter airway were calculated [8].

\section{Genotyping}

Genotyping in NETT was performed for 122 SNPs in six candidate genes (19 in EPHX1, five in SFTPB, 21 in TGFB1, 64 in SERPINE2, seven in GSTP1, six in ADRB2), including upstream and downstream chromosomal regions. We used pairwise linkage disequilibrium (LD)-tagging in Tagger [9] with a minimum minor allele frequency of 0.10 and an $r^{2}$-threshold of 0.9 based on genotype data in Phase II of the HapMap Project (www.hapmap.org), in order to select a panel of SNPs to capture the LD information in each gene. Additional SNPs were also genotyped, based on previously reported genetic association analyses of COPD-related phenotypes [10-12]. SNPs were genotyped using TaqMan (Applied Biosystems, Foster City, CA, USA) or Sequenom (Sequenom, Inc, San Diego, CA, USA) assays as previously reported [13, 14].

\section{Statistical analysis}

Phenotypes tested for association in NETT included per cent of lung area below -950 HU (LAA950), wall thickness and derived SRWA of 10-mm internal perimeter airways. Multivariate analysis was performed using linear regression models with SAS PROC GLM (SAS Institute, Cary, NC, USA), assuming an additive mode of inheritance, and adjusting for age, sex, weight, pack-yrs of cigarette smoking, and clinical centre. All statistical analyses were performed using SAS (SAS Institute). LD between SNPs was assessed using the Haploview program [15].

\section{RESULTS}

\section{Demographic characteristics}

In NETT, the mean age of 379 subjects who had quantitative CT emphysema and/or airway wall measurements was $67.5 \mathrm{yrs}$, and mean FEV1 \% pred was $24.9 \%$ (table 1). Emphysema data were available from 358 subjects, and airway data were available from 338 subjects. The mean (range) number of airways analysed per subject was 12 (4-23).

\section{Association analysis with CT phenotypes in NETT}

As shown in table 2, three SNPs in EPHX1 (rs2854450, rs3738042 and rs1009668), five SNPs in SERPINE2 (rs6734100, rs729631, rs975278, rs6436449 and rs7608941) and one SNP in GSTP1 (rs11227844) were significantly associated with LAA950. Five SNPs in TGFB1 (rs1800469, rs1800470, rs1800471, rs11083616 and rs11466321), two SNPs in EPHX1 (rs2854450 and rs3753658), one SNP in SERPINE2 (rs6436459) and two SNPs in ADRB2 (rs1042717 and rs1042718) were associated with airway wall phenotypes (table 3). Statistical analysis showed similar results for airway wall thickness and SRWA. Among the significantly associated SNPs in table 2, rs729631 and rs975278 in SERPINE2 (pairwise $\mathrm{r}^{2}=97 \%$ ) were in strong LD. Among the significantly associated SNPs in table 3, rs1800470 and rs11083616 (pairwise $\mathrm{r}^{2}=95 \%$ ), rs1800471 and rs11466321 (pairwise $\mathrm{r}^{2}=94 \%$ ) in TGFB1, and rs1042717 and rs1042718 (pairwise $\mathrm{r}^{2}=83 \%$ ) in $A D R B 2$ were in strong LD. A list of genotyped SNPs and LD maps is provided in supplementary table 1 and supplementary figures 1-5, and genetic association results of all SNPs are shown in supplementary table 2 (see online supplementary material).

At the gene level, SNPs in EPHX1 and SERPINE2 were associated with both airway wall and emphysema phenotypes; however, only rs2854450 in EPHX1 was associated with both airway and emphysema phenotypes (fig. 1). The minor allele of rs2854450 was associated with less severe emphysema and thinner airway walls.

\begin{tabular}{lc} 
TABLE 1 & $\begin{array}{l}\text { Demographic characteristics of analysed } \\
\text { subjects in the National Emphysema Treatment } \\
\text { Trial (NETT) Genetics Ancillary Study }\end{array}$ \\
Characteristics & NETT \\
\hline Subjects $\mathbf{n}$ & 379 \\
Age yrs & $67.5 \pm 5.8$ \\
Males & $241(64)$ \\
Cigarette smoking pack-yrs & $66.4 \pm 30.5$ \\
Pre-bronchodilator FEV $\mathbf{1} \%$ pred & $24.9 \pm 6.6$ \\
Post-bronchodilator FEV $\mathbf{1} \%$ pred & $28.1 \pm 7.4$ \\
LAA950 \% & $17 \pm 11$ \\
Wall thickness $\mathbf{m m}$ & $1.53 \pm 0.25$ \\
Square root wall area $\mathbf{~ m m}$ & $4.6 \pm 0.5$ \\
\hline
\end{tabular}

Data are presented as mean \pm SD or $\mathrm{n}(\%)$, unless otherwise stated. FEV 1 : forced expiratory volume in $1 \mathrm{~s}$; \% pred: \% predicted. LAA950: lung area below $-950 \mathrm{HU}$. In NETT, emphysema data were available in 358 subjects and airway wall data (wall thickness and square root wall area) were available in 338 subjects. 


\begin{tabular}{|c|c|c|c|c|c|}
\hline \multirow{3}{*}{$\begin{array}{l}\text { TABLE } 2 \\
\text { Gene }\end{array}$} & \multicolumn{5}{|c|}{$\begin{array}{l}\text { Genetic association results of quantitative } \\
\text { emphysema measurements in subjects in the } \\
\text { National Emphysema Treatment Trial Genetics } \\
\text { Ancillary Study }\end{array}$} \\
\hline & \multirow[t]{2}{*}{ SNP } & \multirow[t]{2}{*}{ Location } & \multirow[t]{2}{*}{ MAF } & \multicolumn{2}{|c|}{ LAA950 } \\
\hline & & & & $\beta$ & $p$-value \\
\hline \multirow[t]{3}{*}{ EPHX1 } & rs2854450 & Promoter & 0.21 & -1.6 & 0.04 \\
\hline & rs3738042 & Promoter & 0.32 & 1.3 & 0.05 \\
\hline & rs1009668 & Exon & 0.11 & 3.3 & 0.002 \\
\hline \multirow[t]{5}{*}{ SERPINE2 } & rs6734100 & Intron & 0.14 & -2.5 & 0.01 \\
\hline & rs729631 & Intron & 0.19 & -2.0 & 0.02 \\
\hline & rs975278 & Intron & 0.19 & -1.6 & 0.05 \\
\hline & rs6436449 & Intron & 0.17 & -2.2 & 0.02 \\
\hline & rs7608941 & Intron & 0.22 & -1.6 & 0.04 \\
\hline GSTP1 & rs 11227844 & 5' flank & 0.09 & 3.2 & 0.003 \\
\hline
\end{tabular}

SNP: single nucleotide polymorphism; MAF: minor allele frequency; LAA950: per cent of lung area below -950 HU. Each model was analysed assuming an additive mode of inheritance adjusting for age, sex, weight, pack-yrs of smoking and clinical centre. SNPs with $p$-values $\leqslant 0.05$ are shown.

\section{DISCUSSION}

In this study, SNPs in six genes previously associated with COPD susceptibility were investigated for association with emphysema severity and airway wall thickness. In NETT subjects, three genes included SNPs that were associated with emphysema severity, and four genes included SNPs that were associated with airway wall phenotypes.

There have been several previous reports of genetic associations with emphysema or emphysema distribution. One previous study reported that emphysema severity determined by visual emphysema score was associated with a tumour necrosis factor- $\alpha$ promoter polymorphism in 84 subjects with
COPD [4]. Another study revealed that low attenuation area percentage of the lung differed according to $M M P-9$ genotype only in the upper lung zones [5]. In NETT subjects, variants in EPHX1 and GSTP1 showed significant association with emphysema distribution, suggesting that xenobiotic enzymes may contribute to upper lobe dominant emphysema [6].

In the current study, 358 subjects in NETT were investigated for genetic associations with emphysema. SNPs in EPHX1, GSTP1 and SERPINE2 were associated with emphysema severity. One of the SNPs associated with emphysema in EPHX1 (rs1009668) was previously reported by our group to be associated with bronchodilator responsiveness in NETT [16].

Airway wall measurements from chest $\mathrm{CT}$ scans are being increasingly used and validated as COPD phenotypes [17, 18]. Airway remodelling is associated with airway narrowing and wall thickening in COPD subjects [19] and this can be evaluated non-invasively using CT [2]. In 338 NETT subjects, we assessed genetic associations with airway wall thickness and airway wall area. SNPs in EPHX1, SERPINE2, TGFB1 and $A D R B 2$ were associated with airway wall phenotypes. EPHX1 and SERPINE2 were associated with both emphysema and airway wall phenotypes; GSTP1 was associated only with emphysema, and TGFB1 and ADRB2 were associated only with airway wall phenotypes.

EPHX1 is related to oxidative stress and has been repeatedly associated with COPD susceptibility [10, 20]; however, recently it has also been reported not to be associated with COPD susceptibility in a large number of COPD and control subjects [21]. With CT phenotypes, previously, EPHX1 was associated with upper lobe predominant emphysema distribution in NETT subjects [6]. In the current analysis, SNPs in EPHX1 were associated with both emphysema severity and airway wall phenotypes in NETT subjects. At the SNP level, only one SNP in EPHX1 (rs2854450) was associated with both emphysema and airway wall phenotypes in NETT subjects. This SNP was associated with less emphysema and thinner airway walls,

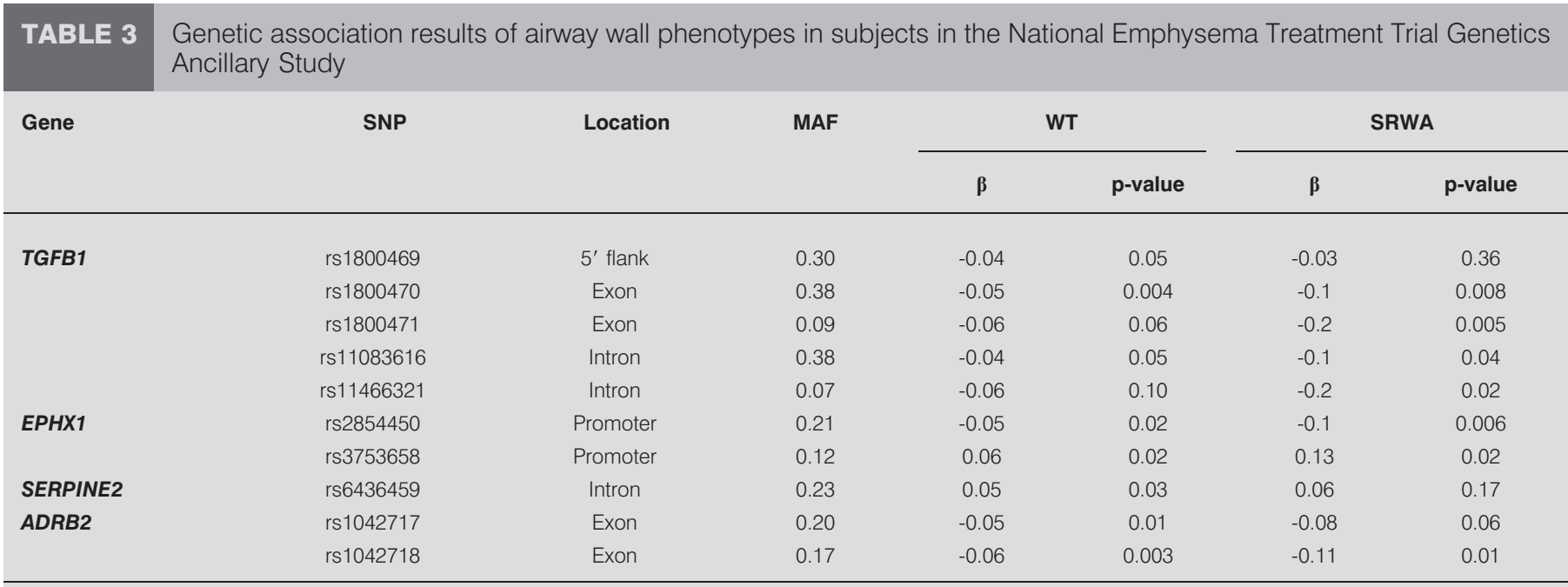

SNP: single nucleotide polymorphism; MAF: minor allele frequency; WT: wall thickness of a hypothetical 10-mm luminal perimeter airway; SRWA: square root of wall area of a hypothetical 10-mm luminal perimeter airway. Each model was analysed assuming an additive mode of inheritance and adjusting for age, sex, weight, pack-yrs of smoking and clinical centre. 


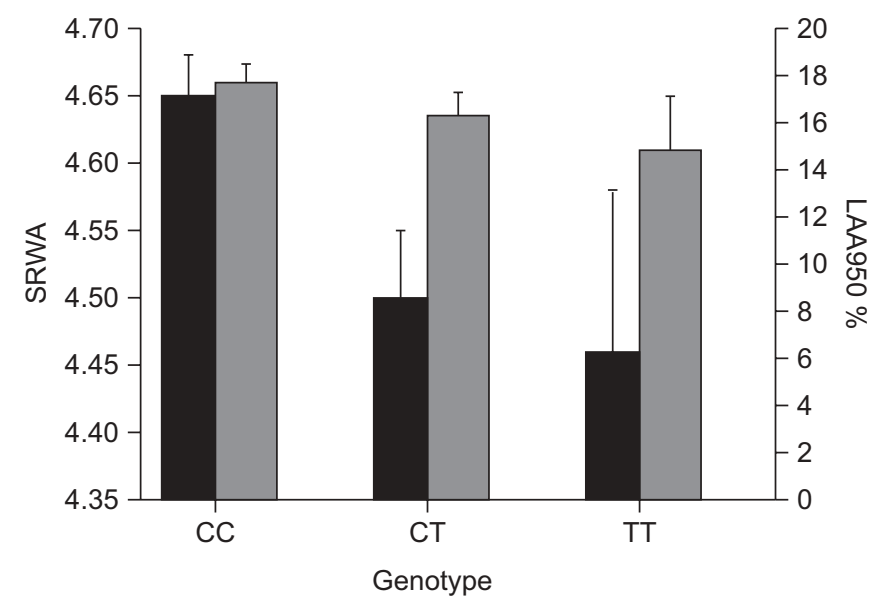

FIGURE 1. Square root of wall area (SRWA) of a hypothetical 10-mm internal perimeter airway and \% of lung area below -950 HU (LAA950) by genotype at rs285540 single nucleotide polymorphism (SNP) in EPHX1 in National Emphysema Treatment Trial subjects. Mean \pm SEM for SRWA ( $\mathbf{\square})$ and LAA950 (四) are shown. CC, CT and TT represent the genotypes at this SNP. CC: $n=206 ; C T: n=101 ; T T: n=17$.

although there was negative overall correlation between emphysema severity and airway wall thickness in NETT subjects [8]. It is not clear whether that promoter SNP has a direct functional effect; it is more likely that it is in LD with a functional variant or variants in EPHX1.

Transforming growth factor (TGF)- $\beta 1$ was previously reported to be associated with COPD by our research group [11]. TGF$\beta 1$ has been suggested to be involved in airway remodelling in COPD [22, 23]. In 85 asthma subjects, serum TGF- $\beta 1$ levels were associated with a promoter polymorphism in TGFB1, but there was no association between airway wall thickness and TGFB1 genetic polymorphisms [24]. However, in 27 subjects with asthma, sputum TGF- $\beta 1$ levels were associated with airway wall area corrected by body surface area [25]. In the present study, several SNPs in TGFB1 were associated with airway wall thickness in the NETT cohort. Among SNPs with significance, rs1800469 and rs1800470 were previously reported to be associated with COPD [11] and respiratory symptom severity phenotypes [13] by our group.

One SNP (rs1042713) of ADRB2 was recently reported to be associated with airway lumen area in Korean COPD subjects [26]; however, ADRB2 SNPs rs1042717 and rs1042718 were associated with airway wall thickness in the current study. The optimal airway phenotypes to reflect airway remodelling or COPD severity have not been determined.

It is interesting that some xenobiotic enzymes (EPHX1) and protease-related genes (SERPINE2) were associated with both emphysema and airway phenotypes, while possible airway remodelling-related genes (TGFB1 and ADRB2) were associated only with airway wall phenotypes. There may be different mechanisms of pathogenesis for emphysema and airway remodelling. Although we recognise that our association results will need to be replicated in larger populations and different ethnic groups, genetic studies may provide insight into these pathogenetic mechanisms. For example, TGF- $\beta 1$ is known to have a role in immune response, cell growth and tissue repair [11]. Although, in animal models, TGFB1 was important in the development of emphysema [27], in our study, this gene was associated with airway phenotypes in a severe COPD cohort suggesting that TGFB1 may be associated with airway remodelling. ADRB2 was reported to be associated with COPD susceptibility and $\beta_{2}$-agonist responsiveness [8]. Although this gene may be related to bronchodilation, it may also have a role in airway remodelling.

The statistical significance of the genetic associations with CT phenotypes in this study was not stronger than previous reports from our group using functional impairment [13] and emphysema distribution [6] phenotypes. It is possible that the currently available emphysema and airway phenotypes do not optimally capture these disease processes. We analysed SNPs in six candidate genes, and it is likely that many other genes also contribute to emphysema and airway disease pathogenesis.

There are several limitations to this study. Subjects were recruited from multiple clinical centres with different CT scanners that may affect the CT parameters. Although we adjusted for clinical centre in our association analysis, residual bias could still exist. We analysed SNPs in six genes, and multiple statistical comparisons are a potential concern. In this initial analysis of genetic determinants of CT-related phenotypes, we did not correct association p-values for multiple statistical testing. We acknowledge that many of the SNPs which showed modest significance with p-values less than a nominal threshold of 0.05 may be false positives. Although the optimal approach to adjust for multiple testing is not clear, multiple statistical testing may be less of a problem since most of these genes have already been associated with COPD susceptibility in multiple populations. In addition, replication of our results in other populations will be required.

The NETT population is a US-based cohort of patients with advanced COPD and a clinical and subjective imaging diagnosis of emphysema. These subjects were screened specifically for participation in a clinical trial of lung volume reduction surgery. They are a predominantly emphysematous subgroup of COPD cases. This may have limited the detection power of the genetic data for the airway phenotypes. However, we previously found that lung function was associated with airway wall thickness in NETT subjects [8], and we now report genetic associations with airway wall thickness in this same population. Thus, airway wall phenotypes may contribute to airflow obstruction even in subjects with severe emphysema.

In conclusion, one SNP in EPHX1 was associated with both emphysema and airway wall thickness using CT in a severe COPD population. Significant associations with emphysema and airway wall area individually were found for SNPs in several other candidate genes. Different genetic determinants may influence airway remodelling and emphysema development. Further work will be required to confirm the genetic determinants of emphysema and airway disease.

\section{SUPPORT STATEMENT}

This manuscript is subject to the NIH Public Access Policy (http:// publicaccess.nih.gov/). This study was funded by the following grants. NIH R01 HL075478, P01 HL083069, R01 HL084323, U01 HL089856, K23HL089353, and the Parker B. Francis Foundation. Funding was also provided by GlaxoSmithKline. 


\section{STATEMENT OF INTEREST}

Statements of interest for E. Hoffman and E.K. Silverman, and for the study itself can be found at www.erj.ersjournals.com/site/misc/ statements.xhtml

\section{ACKNOWLEDGEMENTS}

The authors would like to sincerely thank the manuscript reviewers for helpful suggestions.

\section{REFERENCES}

1 Rabe KF, Hurd S, Anzueto A, et al. Global strategy for the diagnosis, management, and prevention of chronic obstructive pulmonary disease: GOLD executive summary. Am J Respir Crit Care Med 2007; 176: 532-555.

2 de Jong PA, Muller NL, Pare PD, et al. Computed tomographic imaging of the airways: relationship to structure and function. Eur Respir J 2005; 26: 140-152.

3 Patel BD, Coxson HO, Pillai SG, et al. Airway wall thickening and emphysema show independent familial aggregation in chronic obstructive pulmonary disease. Am J Respir Crit Care Med 2008; 178: 500-505.

4 Sakao S, Tatsumi K, Igari $\mathrm{H}$, et al. Association of tumor necrosis factor- $\alpha$ gene promoter polymorphism with low attenuation areas on high-resolution CT in patients with COPD. Chest 2002; 122: 416-420.

5 Ito I, Nagai S, Handa T, et al. Matrix metalloproteinase-9 promoter polymorphism associated with upper lung dominant emphysema. Am J Respir Crit Care Med 2005; 172: 1378-1382.

6 DeMeo DL, Hersh CP, Hoffman EA, et al. Genetic determinants of emphysema distribution in the National Emphysema Treatment Trial. Am J Respir Crit Care Med 2007; 176: 42-48.

7 National Emphysema Treatment Trial Research Group, A randomized trial comparing lung-volume-reduction surgery with medical therapy for severe emphysema. N Engl J Med 2003; 348: 2059-2073.

8 Kim WJ, Silverman EK, Hoffman E, et al. CT metrics of airway disease and emphysema in severe COPD. Chest 2009; 136: 396-404.

9 de Bakker PI, Yelensky R, Pe'er I, et al. Efficiency and power in genetic association studies. Nat Genet 2005; 37: 1217-1223.

10 Hersh CP, DeMeo DL, Lange C, et al. Attempted replication of reported chronic obstructive pulmonary disease candidate gene associations. Am J Respir Cell Mol Biol 2005; 33: 71-78.

11 Celedon JC, Lange C, Raby BA, et al. The transforming growth factor- $\beta 1$ (TGFB1) gene is associated with chronic obstructive pulmonary disease (COPD). Hum Mol Genet 2004; 13: 1649-1656.

12 DeMeo $\mathrm{D}$, Mariani $\mathrm{T}$, Lange $\mathrm{C}$, et al. The SERPINE2 gene is associated with chronic obstructive pulmonary disease. Am J Hum Genet 2006; 78: 253-264.
13 Hersh CP, DeMeo DL, Lazarus R, et al. Genetic association analysis of functional impairment in chronic obstructive pulmonary disease. Am J Respir Crit Care Med 2006; 173: 977-984.

14 Hersh C, DeMeo D, Reilly J, et al. Xenobiotic metabolizing enzyme gene polymorphisms predict response to lung volume reduction surgery. Respir Res 2007; 8: 59.

15 Barret JC, Fry B, Maller J, et al. Haploview: analysis and visualization of LD and haplotype maps. Bioinformatics 2005; 21: 263-265.

16 Kim WJ, Hersh CP, DeMeo DL, et al. Genetic association analysis of COPD candidate genes with bronchodilator responsiveness. Respir Med 2009; 103: 552-557.

17 Hasegawa M, Nasuhara Y, Onodera Y, et al. Airflow limitation and airway dimensions in chronic obstructive pulmonary disease. Am J Respir Crit Care Med 2006; 173: 1309-1315.

18 Nakano Y, Muro S, Sakai H, et al. Computed tomographic measurements of airway dimensions and emphysema in smokers. Correlation with lung function. Am J Respir Crit Care Med 2000; 162: 1102-1108.

19 James AL, Wenzel S. Clinical relevance of airway remodelling in airway diseases. Eur Respir J 2007; 30: 134-155.

20 Smith CA, Harrison DJ. Association between polymorphism in gene for microsomal epoxide hydrolase and susceptibility to emphysema. Lancet 1997; 350: 630-633.

21 Chappell S, Daly L, Morgan K, et al. Genetic variants of microsomal epoxide hydrolase and glutamate-cysteine ligase in COPD. Eur Respir J 2008; 32: 931-937.

22 Takizawa H, Tanaka M, Takami K, et al. Increased expression of transforming growth factor- $\beta 1$ in small airway epithelium from tobacco smokers and patients with chronic obstructive pulmonary disease (COPD). Am J Respir Crit Care Med 2001; 163: 1476-1483.

23 Churg A, Tai H, Coulthard T, et al. Cigarette smoke drives small airway remodeling by induction of growth factors in the airway wall. Am J Respir Crit Care Med 2006; 174: 1327-1334.

24 Ueda $\mathrm{T}$, Niimi A, Matsumoto $\mathrm{H}$, et al. TGFB1 promoter polymorphism C-509T and pathophysiology of asthma. J Allergy Clin Immunol 2008; 121: 659.

25 Yamaguchi M, Niimi A, Matsumoto $H$, et al. Sputum levels of transforming growth factor- $\beta 1$ in asthma: Relation to clinical and computed tomography findings. I Investig Allergol Clin Immunol 2008; 18: 202-206.

26 Kim WJ, Oh Y-M, Sung J, et al. CT scanning-based phenotypes vary with ADRB2 polymorphisms in chronic obstructive pulmonary disease. Respir Med 2009; 103: 98-103.

27 Morris DG, Huang X, Kaminski N, et al. Loss of integrin alpha(v)beta6-mediated TGF-beta activation causes Mmp 12dependent emphysema. Nature 2003; 22: 169-173. 